Nonlinear Processes in Geophysics (2002) 9: 37-49

Nonlinear Processes

in Geophysics

CE European Geophysical Society 2002

\title{
Low-frequency variability in idealised GCM experiments with circumpolar and localised storm tracks
}

\author{
W. Müller ${ }^{*}$, R. Blender ${ }^{1}$, and K. Fraedrich ${ }^{1}$ \\ ${ }^{1}$ Universität Hamburg, Meteorologisches Institut, Hamburg, Germany \\ *Present address: MeteoSwiss, Zürich, Switzerland
}

Received: 1 March 2001 - Revised: 21 June 2001 - Accepted: 23 June 2001

\begin{abstract}
Idealised global circulation model simulations with circumpolar and localised (one and two) storm tracks are re-analysed to determine scaling, intermittency and phase-space structures. In a hundred year experiment with a circumpolar storm track, the spectrum $S(f)$ of the first principal component of the zonal wind fluctuations shows the following power law regimes: (a) a short-term memory between $f^{-4}$ and $f^{-2}$ up to 50 days and (b) a long-term memory $f^{-1}$ from 50 to 400 days and $f^{-0.24}$ beyond 400 days, similar to observed maritime single station near-surface air temperature data. In the presence of localised storm tracks, the wave number two dominates the dynamics and a long-term memory cannot be detected. The recurrence plot is introduced as a novel tool to comprehensively visualise the evolution of the dynamical system in terms of state separations (distances) in phase space. The patterns allow for a qualitative interpretation of the underlying local phenomena in phase space, such as waves, analogs, extremes, and global regimes. Attractor dimensions are, in general, larger than 10, but they appear to be lower in the wave-dominated regimes of the double storm track experiment.
\end{abstract}

\section{Introduction}

The variability observed in the atmosphere covers a wide range of time scales. In addition to the storm tracks, which occur in the frequency band below 10 days, weather regimes and the related blocking events are observed with 10 to 50 day periods. Interannual variability is generally attributed to the slow components of the climate system (hydrosphere or cryosphere). On time scales from days to decades, a power law behaviour corresponding to the spectrum $f^{-0.3}$ has been observed in near-surface temperature anomalies at stations distributed worldwide (Koscielny-Bunde et al., 1998). Fraedrich and Larnder (1993) detected different scal-

Correspondence to: K. Fraedrich (fraedrich@dkrz.de) ing regimes in European precipitation spectra and, in particular, a $f^{-0.7}$ spectrum for periods in the range of $>3$ years.

Variability ranging from short to decadal time scales also occurs in a purely dynamical global circulation model of the atmosphere which, without zonal asymmetry (James and James, 1989, 1992), generates a circumpolar storm track, as represented by the Southern Hemispheric atmospheric flow. A physical mechanism inducing long-period variability has been demonstrated as a wobbler-type regime, describing the interaction between the life cycles of cyclones and the zonal mean flow (James et al., 1994; James and Dodd, 1996). The zonally averaged mean flow patterns propagate slowly poleward through the mid-latitudes with a velocity of $0 \ldots 2 \mathrm{~m} / \mathrm{s}$ (observed a half a century ago by Riehl et al., 1950), and create intermittent behaviour on a wide range of time scales.

To investigate the origin of atmospheric variability of the Northern Hemisphere, localised single and double storm tracks have been simulated by Frisius et al. (1998) and Franzke et al. (2000) with a global circulation model forced by anomalies superimposed on the zonally symmetric heating. The vertically averaged stream function shows various kinds of low-frequency variability with a prominent retrograde wave. Its period near 50 days has been confirmed by observations in the northern Pacific (Kushnir, 1987 and others), and the underlying physical mechanism has been identified as a spatial resonance regime (Franzke et al., 2000), which has also been found in a single layer ocean model (Sura et al., 2000). So far, the analyses of low-frequency variability modelled by simple GCMs are based on standard statistics and physical methods, whereas more sophisticated nonlinear systems analysis methods have not yet been applied.

The aim of this paper is to analyse the low-frequency variability in simplified GCM simulations with circumpolar and localised storm tracks using linear and nonlinear methods that have been, in part, recently developed. The methods used here are (a) spectral and correlation analysis methods to unfold scaling and intermittency and (b) phase-space analysis methods to describe regimes and dimensions. The data 
analysed are time series of the first two principal components of the zonal mean zonal wind (for the circumpolar storm track) and the vertically averaged barotropic stream function (for the localised storm tracks), which have been used to interpret the storm track experiments and to demonstrate low-frequency variability. The outline of the paper is as follows: the model experiments and methods of analysis are introduced in Sect. 2 (and Appendix) and the results are presented in the Sects. 3 and 4. Summary and discussion conclude the analysis.

\section{Idealised experiments and methods of analysis}

The atmospheric variability to be analysed is generated by a global atmospheric circulation model (PUMA, Portable University Model of the Atmosphere, Fraedrich et al., 1998), which is based on the Reading Simplified General Circulation Model (SGCM, Hoskins and Simmons, 1975) and has been further developed in Hamburg. The dynamical core of PUMA is equivalent to GCMs, but dissipative and diabatic processes are parameterised by linear friction and heating; moisture and orographic effects are not included. The model is able to efficiently reproduce the observed circulation and its variability with $\mathrm{T} 21$ wave number truncation $\left(\approx 5^{\circ} \times 5^{\circ}\right)$ on five $\sigma$-levels. It can be used for detailed analyses in prescribed forcing environments. These are represented by diabatic heating $Q=\left(T_{R}-T\right) / \tau_{R}$ with a restoration temperature field $T_{R}$, forcing the temperature $T$ on the time scale $\tau_{R}$. Each model experiment has a duration of 101 years, with the first year being omitted. The analysed experiments have the following configurations of the restoration temperature fields $T_{R}$.

\subsection{Circumpolar storm track}

The experiment simulates a circumpolar storm track excited by a zonally symmetric restoration temperature representing the Southern Hemisphere winter. Figure 1a shows the restoration temperature field $T_{R}$ at $900 \mathrm{hPa}$ (the lowest level of the model). Figure $1 \mathrm{~b}$ presents an overview of the dynamic climatology, given by the mean geopotential height z 900 of the $900 \mathrm{hPa}$ surface, and the standard deviation of the band pass filtered $500 \mathrm{hPa}$ geopotential surface (shaded), which is a common measure of storm track intensity. This type of experiment has been evaluated by James and James (1989, 1992) and James et al. (1994), who detected long-period variability in the zonal mean flow which was unexpected in an atmospheric model. The zonal mean zonal flow configuration is described by the first two Empirical Orthogonal Functions (EOFs, Figs. 2a, b) contributing $36 \%$ and $15 \%$ of the variance in the southern hemisphere. EOF1 describes a weakening and meridional shift in the mid-latitude jet, and EOF2 determines the separation between the subtropical and midlatitude jets.

\subsection{Single storm track}

A single localised storm track is excited by a dipole shaped $T_{R}$-anomaly superimposed on the zonally symmetric restoration temperature field. It represents localised heating by the combined effect of the land/ocean contrast simulated under perpetual spring/autumn conditions (Frisius et al., 1998). Figure 1c shows the restoration temperature at $900 \mathrm{hPa}$ with anomalies reaching $50 \mathrm{~K}$, and Fig. 1d shows the mean $\mathrm{z} 900$ field and the storm track. High pressure occurs near the cold anomaly and low pressure occurs downstream at higher latitudes. The dominant space-time variability occurs in the barotropic stream function. Their first two EOFs (Figs. 2c, d) characterise wave-like behaviour with variances of $20 \%$ and $15 \%$ and an approximate $90^{\circ}$ phase shift.

\subsection{Two storm tracks}

A more realistic experiment involves two localised storm tracks, excited by two dipole shaped $T_{R}$-anomalies, representing heating in the two Northern Hemispheric ocean basins, again with perpetual spring/autumn conditions. The restoration temperature at $900 \mathrm{hPa}$ (Fig. 1e) has two $50 \mathrm{~K}$ anomalies at longitudes separated by $180^{\circ}$, which lead to a wave number two pattern in the mean geopotential height and the storm track (Fig. 1f). The global barotropic stream function (Franzke et al., 2000) shows a retrograde wave number two for a 50-day period. The first two EOFs (Figs. 2e, f) are responsible for a $14 \%$ and $10 \%$ variance. With a $90^{\circ}$ phase shift, EOF1 and EOF2 superimpose to a progressing wave pattern.

\subsection{Analysis}

These three idealised experiments are analysed to identify nonlinear behaviour and to search for the underlying mechanisms. The physical and standard statistical analysis tools are supplemented by more recently developed methods of nonlinear systems analysis. Since the early application of nonlinear analysis methods to modelled and observed weather and climate data (Nicolis and Nicolis, 1984; Fraedrich, 1986), the linear and nonlinear methodological tools have improved and their specifications extended.

(a) Scaling analysis (Appendix A): scaling regimes in frequency space are determined by power spectra and detrended fluctuation analysis (DFA, Peng et al., 1994) presenting a fluctuation function $F(T)$ changing with lag $T$. In addition, wavelet analysis reveals nonstationarity in the periodic behaviour (Torrence and Compo, 1998)

(b) Phase-space analysis (Appendix B): nonlinear methods describe the dynamics through geometric properties in phase space, which requires transformation to a time delay coordinate phase space to embed the time series. Recurrence plots present a rather new graphical tool to display nonstationarities, regimes, and regime transitions in terms of distances between states in phase space 

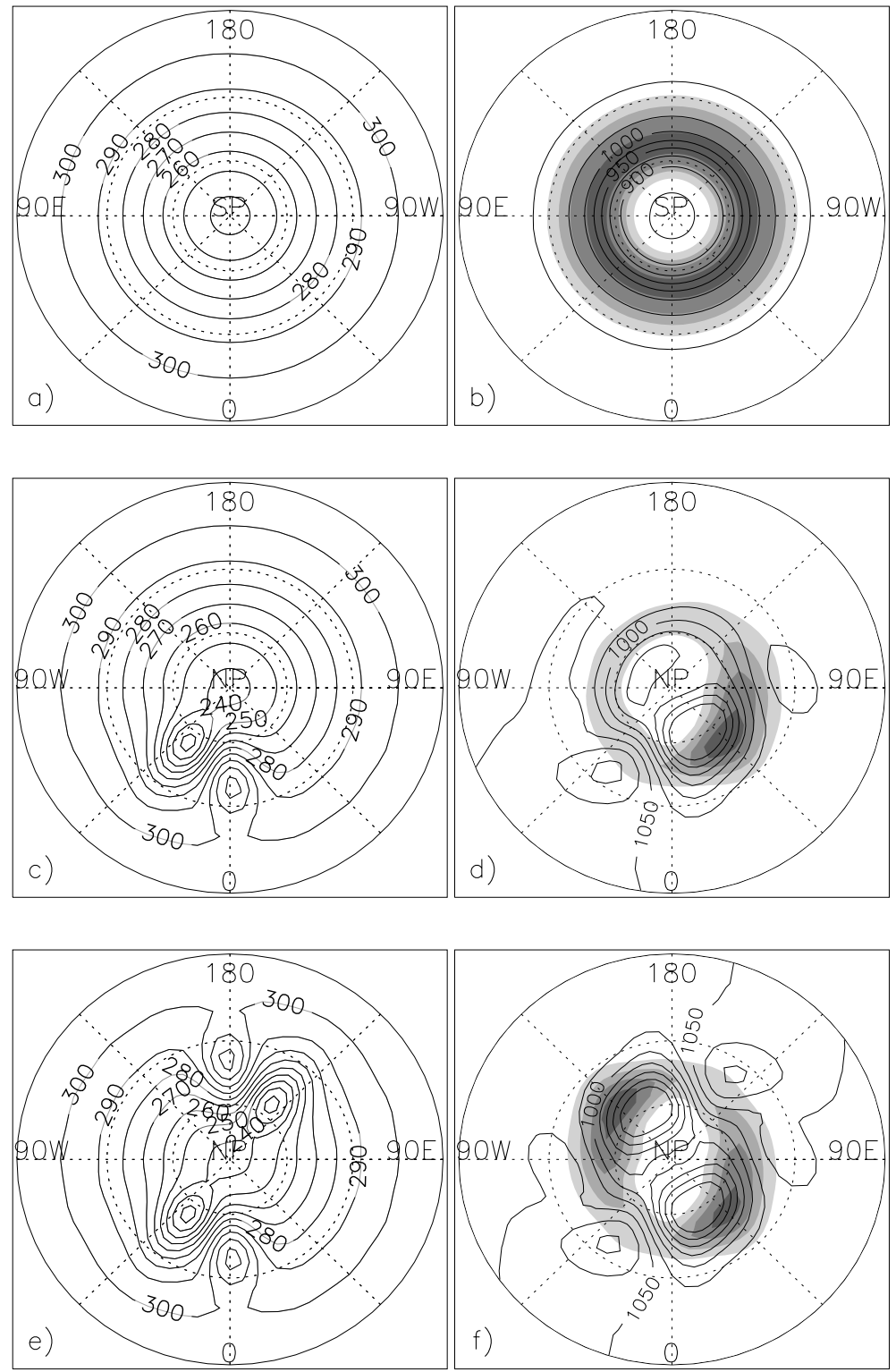

Fig. 1. Climatological setting of the circumpolar storm track (a, b), single storm track (c, d), and the two storm track experiments (e, f). Left panels: restoration temperature field $T_{R}$ at $900 \mathrm{hPa}$ (in $\mathrm{K}$, interval $10 \mathrm{~K}$ ). Right panels: mean geopotential height z 900 at $900 \mathrm{hPa}$ (interval $25 \mathrm{gpm}$ ), and band pass filtered standard deviation of z $500(500 \mathrm{hPa}$, light shade $10 \mathrm{gpm}$, dark shade 40 gpm, interval 10 gpm).
(Eckmann et al., 1987). The calculation of the correlation dimension, which is probably the most popular approach, gives a hint to the number of degrees of freedom of the dynamical system. For reviews of nonlinear analysis methods, (see Abarbanel et al., 1993; Kantz and Schreiber, 1997; Schreiber, 1999). A useful public software package is provided by Hegger et al. (1998).

\section{Circumpolar storm track: a wobbler-regime}

The simplified atmospheric model forced by zonally symmetric heating shows a zonally symmetric storm track with low-frequency variability. James and Dodd (1996) explain this behaviour by the interaction of Rossby waves with the mean zonal wind; wave-driven zonal wind anomalies, denoted as wobblers and visible in the Hovmöller diagram
(James et al., 1994), propagate poleward with a speed up to about $2 \mathrm{~m} / \mathrm{s}$. The summer hemisphere shows well organised coherent structures of the wobblers, while the winter hemisphere presents more disrupted and intense patterns.

\subsection{Scaling regimes}

\subsubsection{Spectrum}

A spectral analysis of the 100 year principal component time series, PC1 and PC2 (Fig. 3a), associated with the spatial EOF-patterns (Figs. 2a, b) exhibits power spectra with three different scaling regimes. For high frequencies up to periods of about $T \approx 20$ days, the spectra $S(f)$ decay rapidly with increasing frequency. The transition at periods of $T=20$ days lies in the range of the characteristic life time of wobblers during the winter season (James and Dodd, 

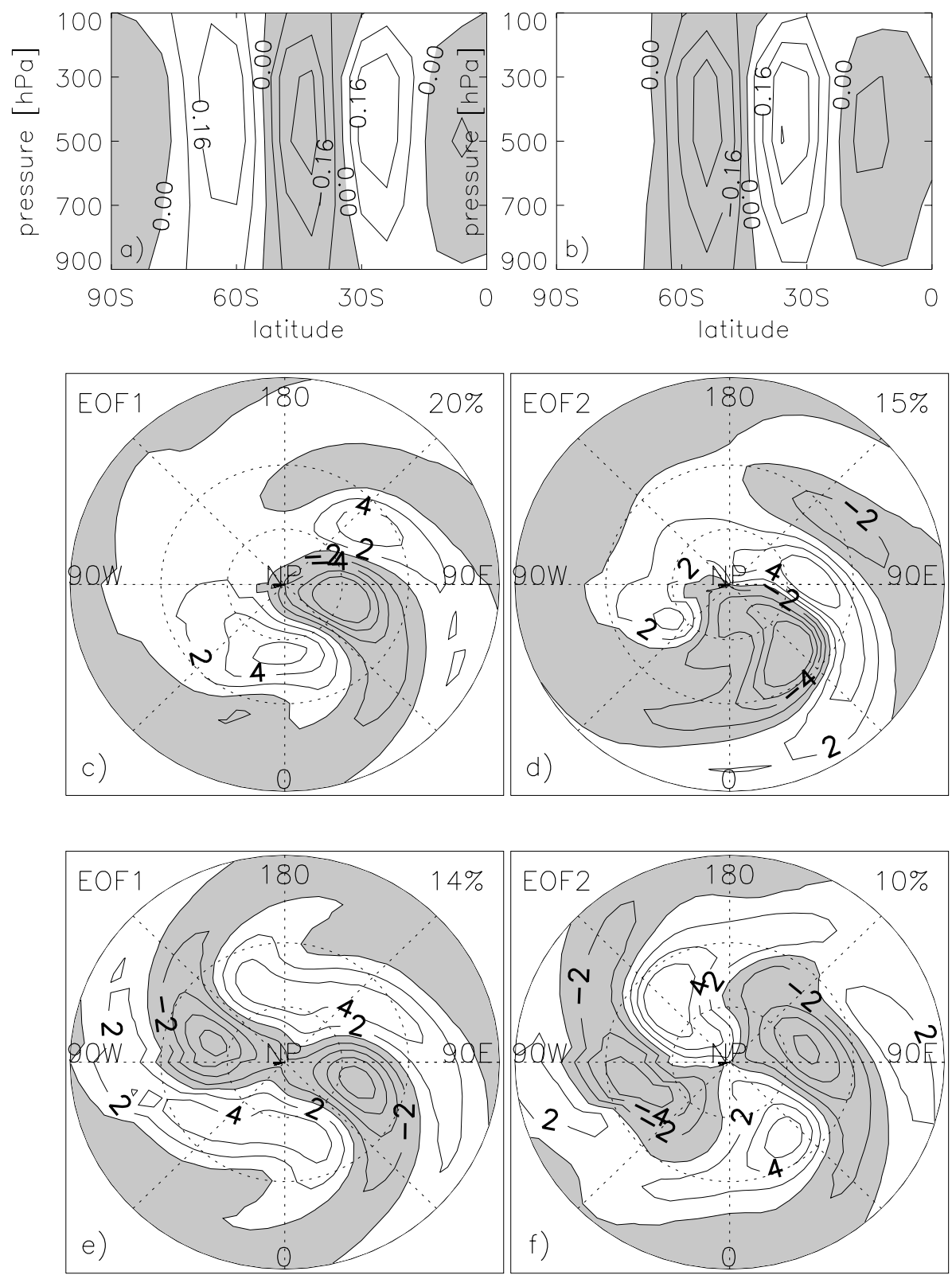

Fig. 2. Empirical orthogonal functions EOF1 and EOF2 (left and right panels) of the circumpolar $(\mathbf{a}, \mathbf{b})$, single storm track (c, d), and the two storm track experiments (e, f): (a, b) The zonal mean zonal wind of the circumpolar storm track, (c, d) the vertically averaged stream functions for a single storm track, and (e, f) for two storm tracks. The EOFs are normalised to unity and, in (c-f), multiplied by 100 . The contour interval is 0.08 in $(a, b)$ and 2 in (c-f), negative values are shaded. The explained variances are included in (cf) at the top right.
1996). The phase spectrum (not shown) indicates that at periods $T>20$ days, PC1 leads PC2. This corresponds to a counterclockwise rotation in the (PC1, PC2)-plane, corresponding to the rotation found by James et al. (1994) for periods slightly above $T>21$ days and, more intensely, within the $40<T<60$ days range. For low-frequencies, the PC2 spectrum reaches a plateau, whereas the PC1 spectrum grows slowly and indicates a low-frequency variability.

\subsubsection{Long-term correlations (detrended fluctuation analysis)}

The long-term correlation of the PC time series is determined by the detrended fluctuation analysis (DFA in Appendix A), showing the fluctuation function $F(T)$ in Fig. $3 \mathrm{~b}$ for PC1 and PC2 (downward shifted for easier visibility). For small periods (lags) $T$, the fluctuation functions grow roughly according to $F \sim T^{\alpha}, \alpha=1.56$. The straight lines (Fig. 3b) are calculated objectively by a linear fit in the interval of the largest possible width under the condition of deviations less than 0.01 between the straight line and the DFA curve. The power $\alpha=1.56$ corresponds to a red noise power law $S \sim f^{-\beta}, \beta=2 \alpha-1 \approx 2$, in the power spectra (Fig. 3a); see Eq. (A2). For longer periods, however, PC1 and PC2 behave differently.

(i) For periods in the intermediate range, $50<T<400$ days, PC1 shows a slope $\alpha \approx 0.95$, which corresponds to the flicker or $1 / f$-noise (see also the linear growth in Fig. 3a). Although the range covers only one order of magnitude in time, this fit in Fig. $3 \mathrm{~b}$ appears to be a 

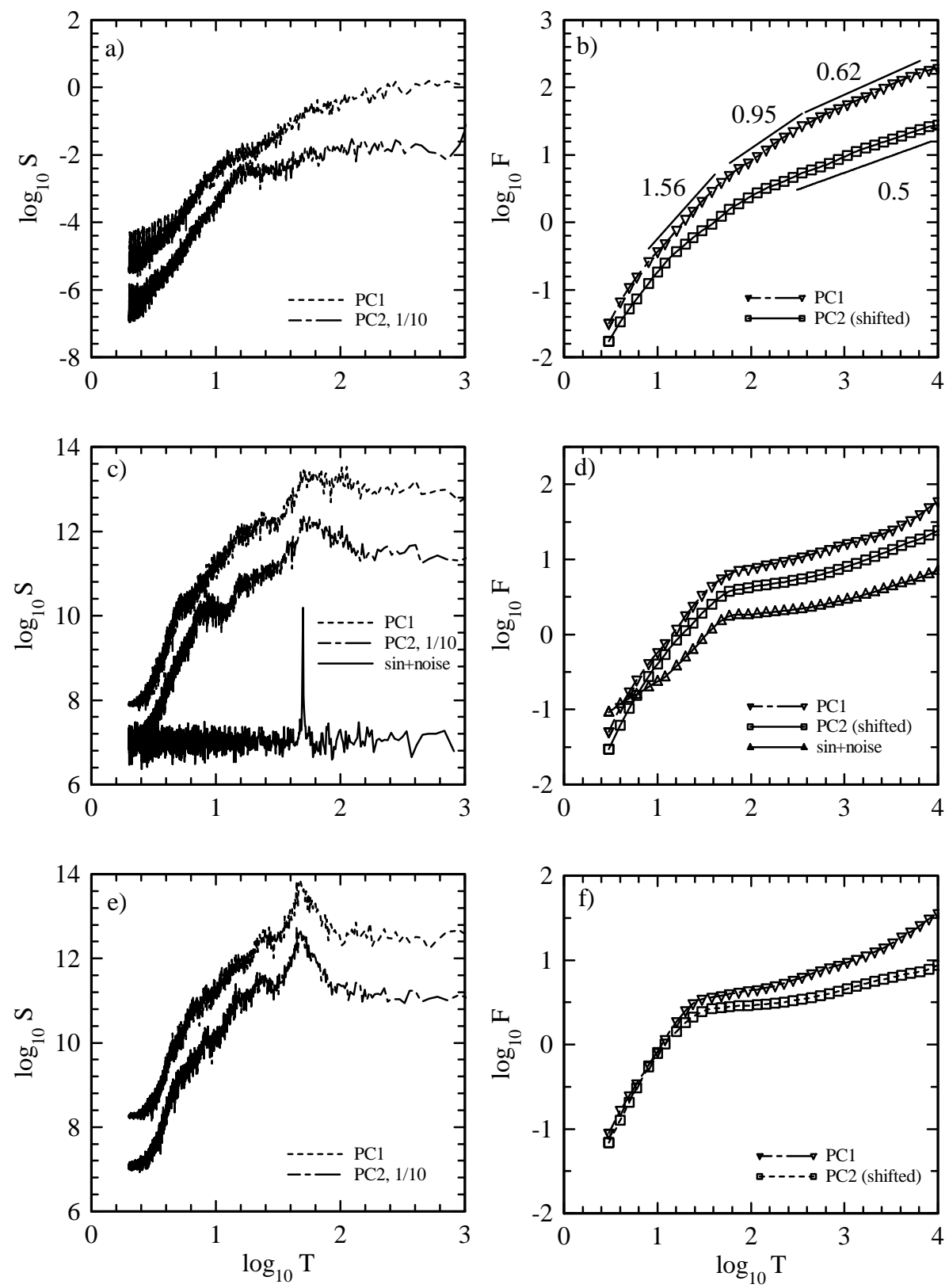

Fig. 3. Power spectra and fluctuation functions (left and right panels) of the circumpolar storm track (a, b), single storm track (c, d), and the two storm track experiments (e, f). Left panels: power spectra $S$ of PC1 (solid) and PC2 (dashed, reduced by $1 / 10$ ) vs. period $T$. Right panels: fluctuation functions $F(T)$ for time lag $T, \mathrm{PC} 1$ (triangles) and PC2 (squares, downward shifted for clarity): (b) Circumpolar storm track with fitted slopes 1.56, 0.95, 0.62, and reference slope 0.5 ; $(\mathrm{c}, \mathrm{d})$ single storm track with noisy sine-oscillation. well localised behaviour and not only a transition phenomenon between high-frequency behaviour and longterm memory. This power law agrees with the results of Yano et al. (2001) for tropical, near-surface temperature observations during the TOGA-COARE experiment.

The origin of the omnipresent $1 / f$ spectrum in nature is an old problem in physics. Recently, Kaulakys and Meškauskas (1998) proposed a simple and analytically solvable model: consider a sequence of equal pulses at times $t_{k}$, created randomly by an autoregressive process of the anomalies, $\theta_{k}=\alpha \theta_{k-1}+\epsilon_{k}$, with the memory $\alpha$ and the white noise $\epsilon_{k}$. The anomalies are $\theta_{k}=t_{k}-t_{k-1}-\bar{t}$ with the mean time $\bar{t}$. This process yields the power spectrum $S(f) \sim f^{-1}$ in a frequency range adjustable by $\alpha$ and the total length of the time series (for further details, see Kaulakys and Meškauskas, 1998). This model provides a possible interpretation in terms of outbursts occurring at a mean separation which is randomised by deviations that follow a simple autoregressive process. Further studies are in progress.

(ii) For longer periods, $T>300$ days, PC1 shows a longrange power law in the fluctuation function, $F(T) \sim$ $T^{\alpha}, \alpha=0.62$, with the correlation $C(T) \sim T^{-\gamma}$, $\gamma=2(1-\alpha)=0.76$; the spectrum is $S \sim f^{-\beta}$, $\beta=1-\gamma=0.24$ (note that this range is not accessible by the conventional power spectrum analysis). The power law in the long-term correlation of PC1 agrees with the findings of Koscielny-Bunde et al. (1998), 
$C(T) \sim T^{-0.7}$, obtained for daily temperature data at 14 stations distributed worldwide. Our result shows that this long-term memory can be reproduced by a simple, uncoupled atmospheric model. However, while some observations show this power law throughout the accessible range, the model and the maritime observations generate this behaviour only for periods higher than 1 year. A more rapid decay in the model (exponent $\gamma=-0.76$ ) compared to the observed $\gamma \approx-0.7$ hints at the model's shorter memory. Note, however, that at higher frequencies, our result deviates from the observations, since the observations show the power law extending over the whole frequency range, with less significance at the high frequencies.

(iii) The PC2-fluctuation spectrum $F(T)$ for longer periods grows like uncorrelated noise with the slope $\alpha \approx 0.5$. This more noisy behaviour of PC2 can be explained by the reduced physical relevance of the second EOF pattern, thus supporting the dynamical interpretation of James et al. (1994).

\subsubsection{Intermittency (wavelets)}

The analysis here and in the following concentrates on PC1, which contains the dominant physical information, while PC2 is either directly related to PC1 (comprising a propagating wave) or dominated by uncorrelated low-frequency noise. The wavelet spectrum (Fig. 4a, Appendix A) shows excitations in the time range of 10 days as an artificial byproduct attributed to the specific shape of the mother wavelet (see the remarks in Appendix A); this is the reason why the power spectrum does not indicate any maximum at this frequency (Fig. 3a). The wobblers at the observed time scales of 100 days (James et al., 1994, in experiments with seven fold symmetry) occur infrequently and are absent in the time mean wavelet spectrum (not shown). Note that the wavelet and the power spectrum (Torrence and Compo, 1998) are normalised differently, so that only their relative values can be compared.

\subsection{Phase-space analysis}

In order to characterise regimes and regime transitions, the PC1 time series is embedded in a higher dimensional phase space spanned by time delay coordinates following the embedding theorem (Appendix B). This leads to a complete reconstruction of the dynamic behaviour of the evolving system, which contrasts the power and wavelet spectrum analyses based on single valued time series.

\subsubsection{Recurrence plot}

The recurrence plot (Eckmann et al., 1987) visualises separations between all pairs of states in the time-evolution of a dynamical system in terms of grey scales or colors (Appendix B). The grey scale is proportional to the distance between the reference state on the diagonal and any past and future state. In this sense, the plot is able to display the system's time evolution after embedding a single-variable time series into a delay coordinate phase space. Here, the dimension is roughly estimated as $d=5$ by the method of nearest false neighbours, and the time delay $\tau=12$ is determined by the first minimum of the average mutual information. Note that the embedded time series is supposed to represent the behaviour of the full system dynamics, although the explained variance is below $100 \%$.

In Fig. 5a, the graphical resolution restricts the display to a variability in the intermediate and longer range, i.e. the smallest visible element is $T \approx 50$ days. A dark (horizontal or vertical) line indicates the presence of a rarely visited point in state space, corresponding to an extremum at the time where the lines cross the diagonal. This is explained by a large distance between this point and the other states visited during the time interval considered. Extreme states are visited rarely (the dark line is interrupted by light points) on time scales of 100 days. Light (horizontal or vertical) lines denote points (at the intersection with the diagonal) that are close to many other states. Light extended squares correspond to quiescent time developments. Wobblers are visible as oscillations (lines with $45^{\circ}$ slopes), with periods of about 100 days, for example, around days 5000 and 5500. The periods are visible as horizontal (or vertical) distances between equal grey scales in a pattern.

\subsubsection{Dimension analysis}

It is of fundamental interest to identify the number of patterns involved in the atmospheric circulation. So far, qualitative methods based on "weather types" and "Grosswetterlagen", of the Northern Hemisphere and the North Atlantic/European sector, and quantitative methods, such as EOF, cluster analysis, fractal dimension and degrees of freedom estimation (Lorenz, 1969; Fraedrich et al., 1994; Toth, 1995) have been applied. These analyses, although uncertain due to the lack of data, lead to rough dimension estimates between 10 and 40 , and hint at a relatively low dimensionality of the largescale circulation.

Here, the PC1 time series is analysed in two different ways: (i) the whole 100-year time range, and (ii) particular time intervals characterised by different (oscillatory) behaviour identified by wavelet and recurrence plot analysis. The latter analysis is motivated by the search for lower dimensional periods, identifiable as regimes employing a dimension calculation. The $D_{2}$-estimation from the slopes of the correlation function (not shown) does not lead to saturation and converge to a limit for an increasing embedding dimension $m=2, \ldots, 20$ (Fig. 5b). Further inspection hints at a linear growth of the fractal dimension with the embedding dimension. The same applies to studies in particular time intervals. All results lead to the conclusion that the dimension $D_{2}>10$.

In summarising the results for the circumpolar storm track, two prominent regimes characterise the variability of the zonal flow: (i) irregular short-period oscillations in the range 

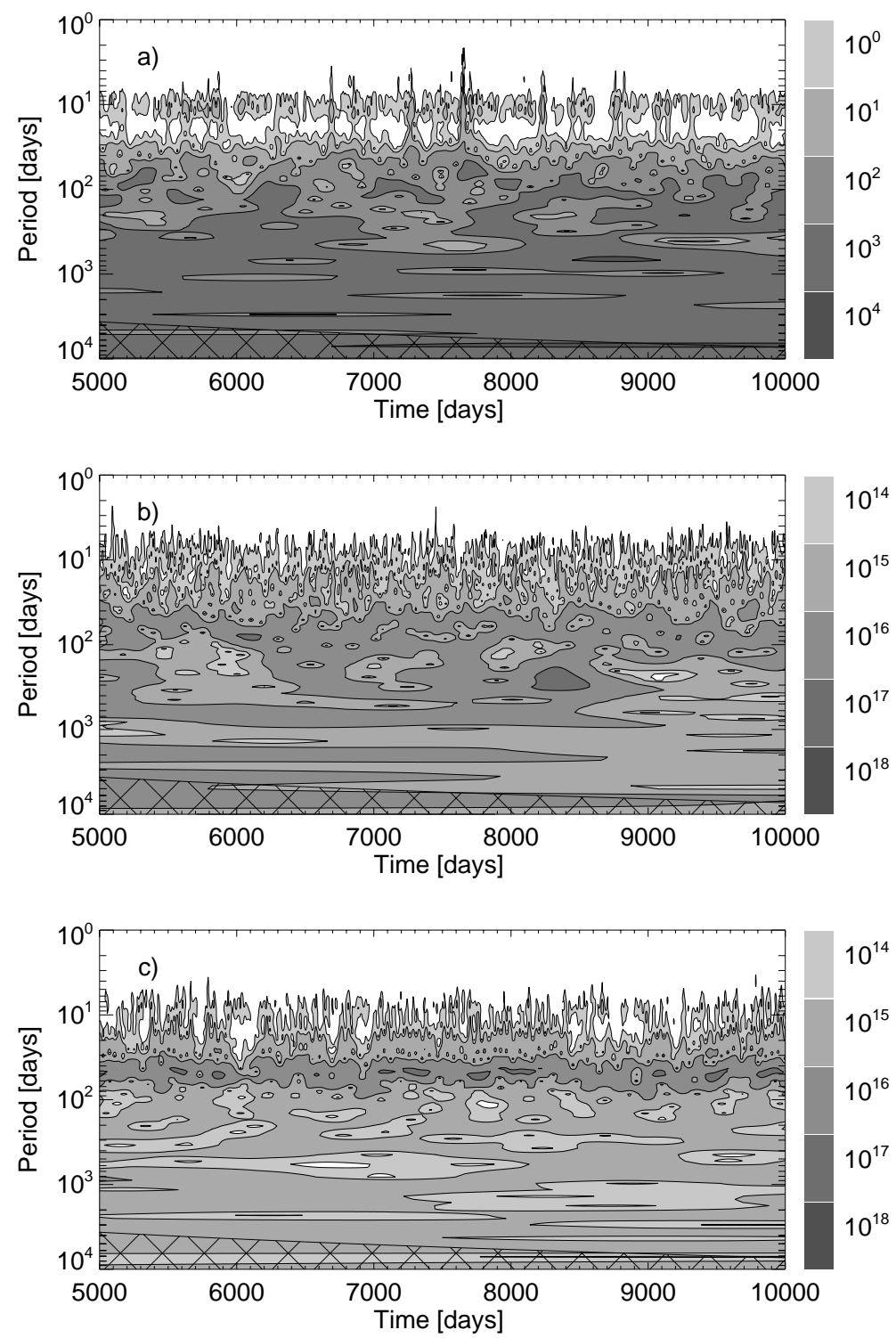

Fig. 4. Wavelet spectra for PC1 in the time interval of 5000-10000 days, and for periods from 1 to $10^{4}$ days: (a) circumpolar storm track, (b) one storm track, and (c) two storm tracks. Values are shown by the grey scale (right), the logarithmic contour interval is unity, and the cone of influence is crosshatched. up to several hundred days and (ii) a broad distribution of long-period oscillations which is evident in the hyperstructure of the recurrence plot. This long-term memory shows two scaling regimes: $f^{-1}$ in 50-400 days and $f^{-0.24}$ beyond 400 days.

\section{Comparison with localised storm tracks: spatial resonance}

Simulations of the atmospheric dynamics on an aqua-planet with zonally symmetric forcing are well suited for experiments with synoptic life cycles, wave mean flow interactions, and low-frequency behaviour under idealised conditions. To analyse the low-frequency behaviour in a more realistic forcing environment, an experiment with two idealised storm tracks, separated by $180^{\circ}$ longitude, is performed; for further comparison, an experiment with a single storm track is in- cluded. Localised storm tracks induce intense low-frequency variability which is associated in PUMA, with a retrograde barotropic wave number two for a 50-day period modulated at the same frequency. The low-frequency variability due to this large-scale wave can be attributed to the generation of anti cyclonic vorticity forcing by synoptic scale vorticity flux divergence (Frisius et al., 1998; Franzke et al., 2000) and has also been found in observations (Kushnir, 1987). Although the magnitude of the wave number two fluctuations is relatively large in the two storm track experiments compared to the observations, the dynamical properties of the flow, the position and intensity of the jets and the storm tracks generated by the $T_{R}$-dipoles compare well with the real atmosphere. The analysis of localised storm tracks is based on the first two EOFs which, in contrast to the circumpolar storm track case, represent the barotropic stream function (Figs. 2c, d: single storm track; Figs. 2e, f: two storm tracks). 

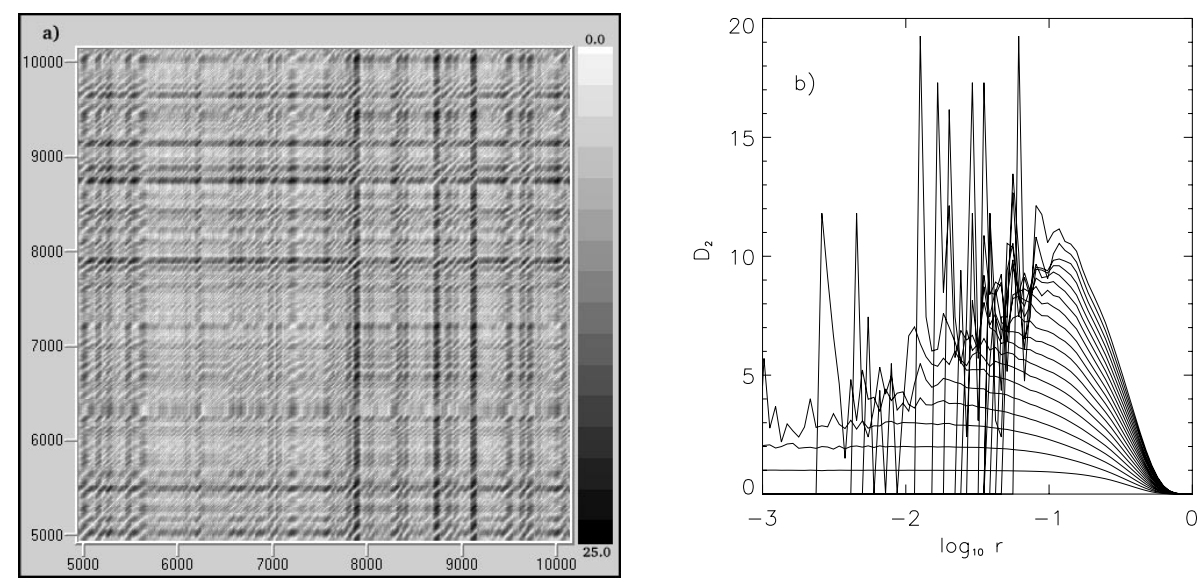

Fig. 5. Circumpolar storm track (PC1) recurrence plot and correlation dimension: (a) recurrence plot sampling the time interval of 500010000 days. Light (dark) grey denotes small (large) distances between the amplitudes at the corresponding times (see label bar). (b) correlation dimensions $D_{2}$ (slopes of $C$ ) vs. radius $r$ for increasing embedding dimensions $m=2, \ldots, 20$ ( $m$ upward).

\subsection{Scaling regimes}

The power spectra of the PC1 and PC2 time series of the storm track experiments (Figs. 3c, e) show pronounced maxima at 50 days; coherence and phase spectra (not shown) of the EOF pairs (Fig. 2c-f) indicate a wave number two westward propagation. The power spectrum of a 50-day oscillation superimposed with noise is included in Fig. 3c for comparison with the DFA result in Fig. 3d. For periods $T<10$ days, which corresponds to the storm track filter, the phase reverses to an eastward propagation and the PCs are less coherent. For a single localised storm track, there is an additional increase in the 7-10 day band variability of PC2 demonstrating the relation of EOF2 (Fig. 2d) to the internal storm track dynamics; higher order EOF patterns (not shown) also display their relation to the mid-latitude baroclinic wave structure.

\subsubsection{Long-term correlations (detrended fluctuation analysis)}

The long-term correlation is determined by the detrended fluctuation analysis (DFA, see Appendix A). Figure 3 displays the fluctuation function $F(T)$ of $\mathrm{PC} 1$ and $\mathrm{PC} 2$ (shifted downward for better display) for one storm track (Fig. 3d) and for two storm tracks (Fig. 3f). To interpret the shape of the fluctuation spectrum $F(T)$ qualitatively, an artificial spectrum is computed for a time series generated by a 50 day oscillation, plus uncorrelated noise. This simple model reveals the same qualitative structure as observed for PC1 and PC2. Note that a pure wave without noise yields a constant fluctuation function $F(T)$ for $T$ larger than the wave period. According to this simple model, the DFA spectra (Figs. 3d, f) indicate a pronounced wave-like structure. Obviously, the long-term behaviour is obscured by the wave in the plot. For very large periods, which are not shown here, the noise of 0.5 -power dominates the sine-oscillation. This hints to a general restriction of the method.

\subsubsection{Intermittency (wavelets)}

The wavelet spectrum (Appendix A) of the single storm track (Fig. 4b) shows a small, homogeneous range of enhanced variability for periods up to 100 days, and sporadic variability occurs for longer periods. The wavelet spectrum of the two storm track runs (Fig. 4c) shows a continuous band of maximum power near the 50-day period. There are certain time intervals, for example, from 7000 to 8000 days, where the power of the spectrum is distinctly higher.

\subsection{Phase-space analysis}

To search for further differences between the circumpolar and the localised storm tracks, the PC1 time series (corresponding to the EOFs in Figs. 2c, e) is embedded in a higher dimensional phase space (Appendix B). The time series are considered in the recurrence plot and analysed by dimension analysis.

\subsubsection{Recurrence plot}

The recurrence plot displays grey scales corresponding to distances between the actual state on the diagonal and all prior (and later) states occurring during the time-evolution. The dynamical system is described by the PC1 time series of the storm track experiments embedded in $d=5$ with a delay time $\tau=10$ days (values estimated as in the circumpolar storm track experiment). In the recurrence plots (Figs. 6a, b), the resolution is adjusted to reveal structures above the time scale of 50 days. For a single storm track, the following results are obtained (Fig. 6a): (a) the amplitudes of the waves appear as contrasts in the grey scale; note that there are intense wave amplitudes in some intervals; (b) sequences of light intervals on horizontal (or vertical) lines are another feature of the single storm track experiment. They indicate near neighbours (i.e. analogs) of the reference state on the diagonal whose persistence is indicated by the length of the lines. 

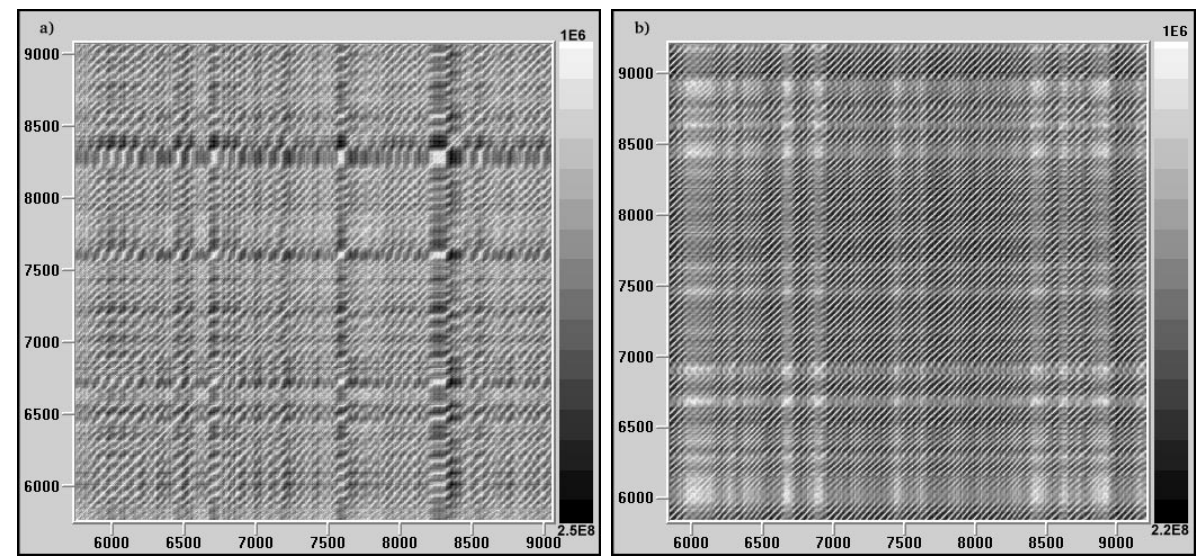

Fig. 6. Recurrence plots of the 6000-9000 days PC1 sample: (a) one storm track and (b) two storm tracks. Light (dark) grey denotes small (large) distances between the amplitudes at the corresponding times (label bar on the right).

Note the difference in the circumpolar storm track where extreme situations (black intervals) are a prominent feature of the time-evolution.

In the two storm track experiments, the 50-day wave occurs as a diagonal slope representing the dominant feature of the whole plot (Fig. 6b). The following points are noted: (a) in contrast to the circumpolar storm track experiment (Fig. 5a), black lines are completely absent, i.e. rarely visited states do not exist; (b) regime transitions do not occur as indicated by the lack of a chequerboard hyperstructure (in contrast to the Lorenz recurrence plot below, Appendix B: Fig. B1a); (c) a few light squares, most pronounced near 6000 days and 200 days in duration, indicate almost stationary states of the model. This type of behaviour prevails from 6000 to 7000 days. Within these squares, the activity of the 50-day wave is strongly suppressed; (d) regular and uninterrupted wave motion occurs between 7000 and 8000 days, which is in agreement with the wavelet spectrum in Fig. 4c. This interval is subjected to dimension analysis and compared to the interval of 6000-7000 days.

The three recurrence plots for the circumpolar and the two localised storm tracks (Figs. 5a, 6a, b) exhibit scaling or long-term memory behaviour and pure oscillations, respectively. The self-similarity, which dominates for the circumpolar storm track, is reduced by introducing a single localised storm track and then vanishes if a second localised storm track enters at a $180^{\circ}$ separation. This is also reflected by the spectral analysis which shows more regular short-period oscillations, while low-frequency scaling is absent.

\subsubsection{Dimension analysis}

The dimension of the localised storm track experiments in the complete 100 yr time interval (not shown) does not converge when increasing the embedding dimension $m$ from 2 to 20 , similar to the circumpolar experiment. Two dynamically distinct time intervals identified in the recurrence plot are chosen for dimension analysis: (a) The interval 60007000 days is characterised by weak intensity of the waves, irregular behaviour and the occurrence of stationary points (Fig. 7a). Not unexpected, there is no saturation in the dimensionality up to the embedding $m=20$ (note that due to the short length of the time series noise prevails at large distances $r$ ). (b) The interval 7000-8000 days is characterised by a regular and intense wave variability. Obviously, the dimension analysis shows a plateau like behaviour for a short spatial range $(r=0.1, \ldots, 0.2)$, suggesting a finite dimensional structure for parts of the trajectory (Fig. 7b).

\section{Summary and discussion}

Circumpolar and localised storm tracks are simulated in a simplified GCM (PUMA) in order to analyse their impact on low-frequency variability of the mid-latitude atmosphere. To obtain localised regions of eddy activity (storm tracks), the model is forced by restoration temperature dipole anomalies acting like nudging fields. Three idealised experiments are distinguished: a circumpolar storm track (aqua-planet), a single storm track, and two storm tracks with $180^{\circ}$ zonal distance. Linear and nonlinear systems analysis methods are applied to the principal components of the first two EOFs, characterising the dominating features of the low-frequency variability. The following results are noted:

The circumpolar storm track is analysed using the first two principal components $\mathrm{PC} 1$ and $\mathrm{PC} 2$ of the EOFs of the zonally averaged zonal wind. The power spectrum of $\mathrm{PC} 1$ shows three regimes associated with the following period ranges:

(i) $T<10$ days: $S \sim f^{-4}$,

(ii) $10<T<50$ days: $S \sim f^{-2}$,

(iii) $50<T<400$ days: $S \sim f^{-1}$.

The model exhibits $1 / f$-noise throughout one order of magnitude. A possible candidate for the explanation are wobblers (mean zonal wind anomalies) serving as pulses in a recently proposed model for the $1 / f$ noise (Kaulakys and Meškauskas, 1998). Application of detrended fluctuation 

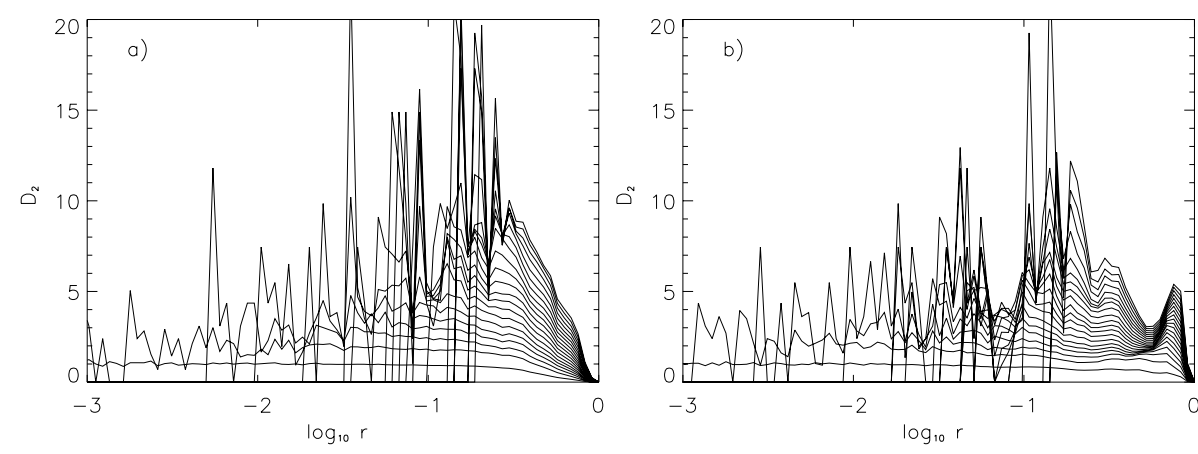

Fig. 7. Two storm tracks correlation dimension $D_{2}$ from PC1 for embedding dimensions $m=2, \ldots, 20$ ( $m$ upward) in the intervals (a) 6000-7000 days, (b) 7000-8000 days.

analysis (DFA) supports the results in the intermediate ranges (ii) and (iii).

Furthermore, the DFA extends the period range to (iv) $T>400$ days: $S \sim f^{-\beta}$. The power $\beta=1-\gamma=0.24$ is associated with the correlation function $C(\tau)=\tau^{-\gamma}$, $\gamma \approx-0.76$, which agrees remarkably well with the longterm correlation discovered in the observed single station data, $\gamma \approx-0.7$ (Koscielny-Bunde et al., 1998). The recurrence plot, which represents the system reconstructed in a delay coordinate phases space, hints at frequent waves and rare extreme events. In addition to irregular short-period oscillations, the plot shows a self-similar hyperstructure with a broad distribution of oscillations in agreement with the power spectra and DFA.

The single storm track experiment is analysed in terms of the principal components of the EOFs of the vertically averaged stream function. A retrograde wave number two with a period of about 50 days is excited and visible in the power and DFA spectra; long-term memory has not been identified. The more realistic two storm track experiment also shows variability dominated by wave number two and, as in the single storm track experiment, long-term memory also seems to be absent or obscured. The recurrence plot visualises stochastically dominated intervals alternating with regimes of wave-like variability of smaller fractal dimension.

Several new nonlinear methods are applied to analyse the low-frequency behaviour of the mid-latitude circulation with different numbers of localised storm tracks. The main themes are for the circumpolar storm track the wobblers, the $1 / f$ power spectrum, a long-term memory as observed in station data, and, for the single and double storm track experiments, stochastic resonance of wave number two. These results are discovered primarily due to the application of rather new methods, such as the wavelet analysis, the recurrence plot, and the detrended fluctuation analysis. The following interpretations are suggested:

(a) The wobblers (James and Dodd, 1996) describe propagating zonal wind anomalies and appear to be responsible for the $1 / f$-power spectrum in an intermediate time range (50-400 days); they have life times of 20 100 days and occur intermittently. The recently pub- lished pulse model (Kaulakys and Meškauskas, 1998) is a promising candidate for the explanation in terms of a simple mechanism and the time scales involved.

(b) The wave number two with the 50-day period, which appears in the single storm track and, more distinctly, in the two storm track experiments, dominates the power and DFA spectra. This prevails up to the highest accessible periods of $10^{4}$ days. Therefore, these unrealistically large wave fluctuations obscure other types of variability. By using the recurrence plot as a tool for the detection of regimes, it is possible to distinguish between intervals of different wave intensity. In intervals with predominantly regular waves, the attractor dimension seems to be considerably lower than for the total range, however, a reliable value cannot be attributed to it.

(c) The long-term memory detected in the idealised GCM runs, similar to those found in observational data (Koscielny-Bunde et al., 1998), is a major result of this analysis. It demonstrates that the observed longterm memory can be reproduced by an uncoupled atmospheric model which is purely dynamical, driven by Newtonian cooling and damped by Rayleigh friction.

In summarising, idealised atmospheric GMC experiments provide a new perspective to interpret the observations in the climate system where the atmosphere interacts with the slower subsystems: the observed long-term behaviour may be a consequence of the nonlinear behaviour of the atmosphere alone, so that the role of the slower components requires further analysis.

\section{Appendix A Spectral and correlation analysis methods}

Spectral analysis: The phase spectrum $\Phi(f)$ and the coherence spectrum $\kappa(f)$ of two time series are determined using the complex cross spectrum $\Gamma(f)$, which is the Fourier transform of the cross-correlation function $\gamma(t)$. Via the decomposition $\Gamma=A \exp (i \Phi)$, the phase $\Phi$ is fixed and the squared 
coherence spectrum can be defined as $\kappa(f)=A^{2} / S_{1} S_{2}$ with the two power spectra $S_{1}(f)$ and $S_{2}(f)$.

Detrended fluctuation analysis (DFA): The calculation of long-term correlations is frequently hindered by noise and nonstationarities. A robust method to overcome these two problems is the detrended fluctuation analysis (DFA), which is able to detect long-term correlations inaccessible to a direct calculation of the correlation function and the spectrum (see Peng et al., 1994). The DFA aims at long-term correlations and spectra via the fluctuations on different time scales. A basic feature of the method is a scale selective correlation analysis. Koscielny-Bunde et al. (1998) applied DFA (among other methods) to observed long-term daily temperature records at 14 stations and found a uniform power law $C(T) \sim T^{-0.7}$ in a wide range of time scales $T$ between ten days and several decades. Note that this type of decay is distinctly different from an exponential decay, since the power law does not possess a characteristic time scale and the decay is much slower. The method works as follows. The correlation function is given for discrete time $T$ in terms of the anomalies $\Delta X_{i}, i=1, \ldots, N$, from which a possible annual cycle is to be subtracted

$C(T)=\left\langle\Delta X_{i} \Delta X_{i+T}\right\rangle=\frac{1}{N-T} \sum_{i=1}^{N-T} \Delta X_{i} \Delta X_{i+T}$.

The integrated anomalies lead to the profile $Y_{n}=$ $\sum_{i=1}^{n} \Delta X_{i}, n=1, \ldots, N$. The time range $N$ is divided in segments $v$ of length $T$, either overlapping $(N-T+1 \mathrm{seg}-$ ments) or non-overlapping ( $N / T$ segments). Since the number of overlapping segments is higher, this type is used below. Fluctuations $F_{v}^{2}(T)$ in all segments $v$ with lengths $T$ are determined by the squared distances between $Y_{n}$ and a fitted straight line, and this line, which is determined for each segment $v$, removes linear trends on the corresponding time scale. The DFA result for the segment mean $F(T)$ can be directly related to the correlation function $C(T)$ in the case of a power law decay

$C(T) \sim T^{-\gamma}, \quad F(T) \sim T^{\alpha}, \quad \alpha=1-\gamma / 2$.

The power spectrum $S(f)$ behaves as $S(f) \sim f^{-\beta}$, $\beta=1-\gamma$. We can distinguish the following cases:

1. $\alpha<1 / 2$ : Antipersistent, primarily due to oscillations.

2. $\alpha=1 / 2$ : No or short-range correlation (e.g. exponential, $\left.C \sim \exp \left(-T / T_{0}\right)\right)$.

3. $1 / 2<\alpha<1$ : Long-range correlation (as in observed temperature records).

4. $\alpha=1: 1 / f$-noise.

5. $\alpha=3 / 2$ : A random walk (brown noise).

For example, an auto-regressive process tends to $F(T) \sim$ $T^{3 / 2}$ for small $T$ (red power spectrum $f^{-2}$ ) and $F(T) \sim$ $T^{1 / 2}$-behaviour for large $T$ (white power spectrum). A single wave shows a rapid growth of $F(T)$ for $T$ below its period, and a constant value above.

Wavelet transform: The wavelet transform determines the intermittent spectral structure of a time series via an extension of the Fourier transform (Torrence and Compo, 1998). The method is able to reveal regime-like periods related to particular frequencies. The time signal $f(t)$ is transformed according to

$$
\begin{array}{r}
f\left(\lambda, t^{\prime}\right)=\int_{\infty}^{\infty} f(t) \Psi_{\lambda, t^{\prime}}(t) d t, \\
\Psi_{\lambda, t^{\prime}}(t)=\frac{1}{\sqrt{\lambda}} \psi\left(\frac{t-t^{\prime}}{\lambda}\right)
\end{array}
$$

with the mother wavelet $\psi$. The parameter $\lambda$ describes scaling and frequency, and $t^{\prime}$ denotes the position analysed. We apply the Morlet wavelet $\psi(t)=\pi^{-1 / 4} \exp \left(i \omega_{0} t-t^{2} / 2\right)$ with $\omega_{0}=6$. The scale $\lambda$ is approximately equal to the Fourier period, $\lambda \approx T$. The wavelet power spectrum $\left|f\left(\lambda, t^{\prime}\right)\right|^{2}$ depends on frequency and time. In the wavelet spectra, the cone of influence marks those regions where the influence of the temporal boundaries hinders the calculations. There is an important but rarely mentioned restriction to the interpretation of wavelet spectra at small scales (Perrier et al., 1995): If the Fourier spectrum is steep at high frequencies, then the wavelet spectrum is strongly influenced by the shape of the mother wavelet. Therefore, in our analysis, the wavelet spectrum cannot be interpreted below 10 days.

\section{Appendix B Phase-space analysis methods}

Employing nonlinear time series analysis, the state of a dynamical system is reconstructed using a single scalar time series $y_{n}$ measured at discrete times $n=1, \ldots, N$. The embedding theorem of Takens (1981) states that the delay time series, embedded in a state space of sufficient (embedding) dimension $m \geq 2 d+1$, represents the coupling of all degrees of freedom and is a proper reconstruction of the complete (unknown) dynamics with dimension $d$. The embedding leads to a multivariate time series vector $\mathbf{y}_{n}$, whose components represent $m$ delay coordinates at the temporal distances $\tau$, $\mathbf{y}_{n}=\left(y_{n}, y_{n+\tau}, \ldots, y_{n+(m-1) \tau}\right)$. The dimension $m$ can be as low as the fractal dimension $D_{2}$ of the attractor of the underlying dissipative dynamical system. For the application of the recurrence plot below, the embedding dimension $m$ is estimated in a first step by the method of False Nearest Neighbours (FNN), which provides a clear and convincing approach (Kennel et al., 1992). The delay $\tau$ is determined here by the first minimum of the average mutual information (Fraser and Swinney, 1986). The embedding is a prerequisite for the recurrence plot.

Recurrence plot: The recurrence plot (Eckmann et al., 1987) displays distances between all pairs of states during the time-evolution of a dynamical system by colours or grey scales. It represents a recurrence matrix $R_{i j}$ for states $\mathbf{y}_{i}, \mathbf{y}_{j}$ at times $i, j$; the matrix is determined for all pairs of states, $R_{i j}=\operatorname{dist}\left(\mathbf{y}_{i}, \mathbf{y}_{j}\right)$, where the distance is given by the sum of 

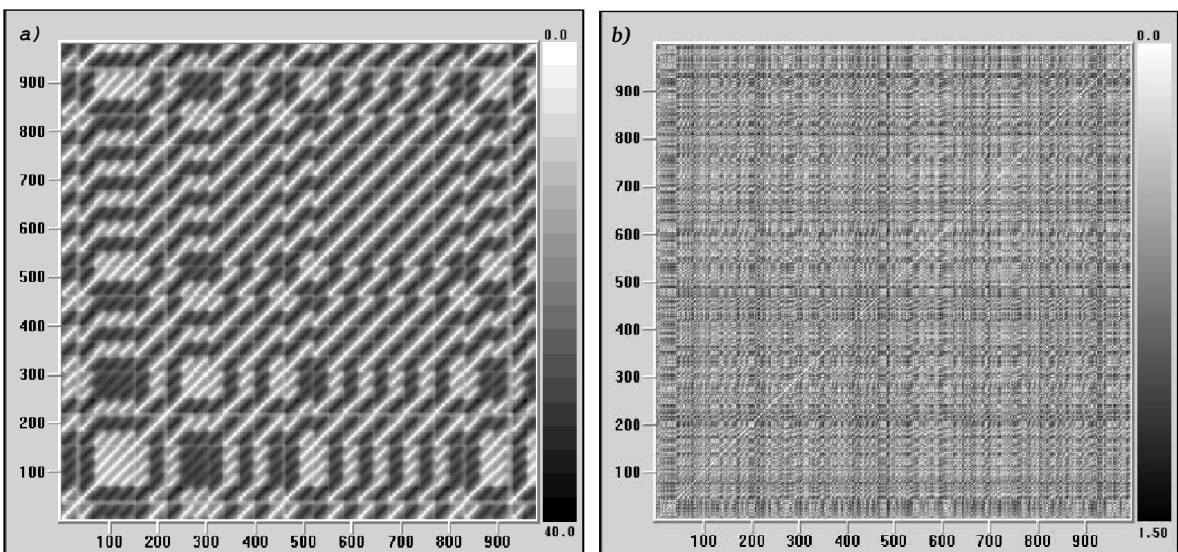

Fig. B1. Recurrence Plots for (a) the Lorenz model (parameters see text), and (b) white noise; light (dark) shades denote small (large) distances of the states in embedding space (see bar at the right). The times are along the ordinate and the abscissa (range 0-1000). squares of the embedding component differences. The matrix values are mapped to a grey scale, where white (black) denotes a vanishing (maximum) distance; by definition, the plot is symmetrical with respect to the diagonal. All plots have a white line of vanishing distance in common, it marks the diagonal where the states are identical. The plot highlights the time-evolution of distances in state space, showing waves, regimes, analogs and extreme states. Two examples illustrate the interpretation of the recurrence plot: the $x$-component of the well-known Lorenz (1963) system with $\sigma=10, b=8 / 3$, and $r=60$ shows the following dynamics (Fig. B1a):

- Amplitude regimes are characterised by extended light and dark rectangles (chequerboard hyperstructure). Within each rectangle, the system remains in one-half - or a regime - of the attractor. A dark rectangle visualises persistent separation from the state reached by crossing a horizontal (or vertical) line with the diagonal. By definition, along the diagonal, only light rectangles are possible.

- The regime transitions are visible as intense dark-light contrasts parallel to the axes. The regular square in the time interval of $550-850$ Lorenz time units contains a sequence of regime transitions between the two halves.

- Oscillations are represented by $45^{\circ}$ slopes within onehalf of the attractor, for example, the light rectangle in the 50-180 Lorenz time unit range.

White noise (Fig. B1b) shows no structure at any time scale. The recurrence plot is able to highlight the following types of dynamic behaviour (these interpretations are motivated by the results of the present study):

- A reference point at a given time is rarely visited during the time-evolution if it is associated with a dark horizontal (vertical) line, since dark grey is a measure of the large distances of this point to the other states visited.
- A light horizontal or vertical line denotes points (at the intersection with the diagonal) that are visited frequently.

- Light regions (approximate squares) correspond to quiescent time developments.

In summarising, nonstationary regime-like behaviour shows up in the recurrence plot as coarse patterns superimposed to the fast fluctuations. Therefore, they are an ideal tool to diagnose the slow variability through the "hyperstructure" patterns. Note that for very high dimensional systems, these plots are, at times, "rather difficult to read" (Schreiber, 1999). In addition to regime diagnostics, recurrence plots are used to identify time regimes of similar dynamic structures which are subjected to further dimension analysis to quantify their static properties.

Attractor dimension: The fractal dimension is determined by the algorithm of Grassberger and Procaccia (1983). First, the correlation integral $C(r)$ of the observed system states $\mathbf{y}_{i}$ in the embedding space is determined

$C(r)=\frac{1}{N^{2}} \sum_{i=1}^{N} \sum_{j=1, j \neq i}^{N} \theta\left(r-\left|\mathbf{y}_{i}-\mathbf{y}_{j}\right|\right)$,

where $\theta(x)$ is the Heaviside step function, $\theta(x \geq 0)=1$, and $\theta(x<0)=0$. The correlation integral counts the number of points that are in the $r$-neighbourhood of a given reference point. On a strange attractor, the correlation integral grows according to a power law, $C(r) \sim r^{D_{2}}$ with the correlation dimension $D_{2}$. This can be determined by the slope in the log-log plot of $C(r), D_{2}=d \log C / d \log r$, in a range restricted by the minimum distance of the points simulated, and by the extent of the attractor. A fractal dimension can be attributed to the attractor if the slope is independent of the embedding dimension $m$. Nonstationarities might hinder a dimension estimation.

Acknowledgements. We like to thank C. Franzke, T. Frisius, E. Kirk, U. Luksch, and F. Lunkeit (all at Hamburg University) for the data and useful discussions. We appreciate the use of the software TISEAN (R. Hegger, H. Kantz, and T. 
Schreiber). Recurrence plots were created by the software Visual Recurrence Analysis (VRA) by E. Kononov available at http://pw1.netcom.com/ eugene. The wavelet analysis was performed by the software of Torrence and Compo (1998) available at http://paos.colorado.edu/research/wavelets. Support by the DAAD (Deutscher Akademischer Austauschdienst) and the Sonderforschungsbereich SFB-512 ("Tiefdruckgebiete und Klimasystem des Nordatlantiks") is acknowledged.

\section{References}

Abarbanel, H. D. I., Sidorowich, J. J., and Tsimring, L. S.: The analysis of observed data in physical systems, Rev. Mod. Phys., 65, 1331-1391, 1993.

Eckmann, J.-P., Kamphorst, S. O., and Ruelle, D.: Recurrence plots of dynamical systems, Europhys. Lett., 4 (9), 973-977, 1987.

Fraedrich, K.: Estimating the dimensions of weather and climate attractors, J. Atmos. Sci., 43, 419-432, 1986.

Fraedrich, K. and Larnder, C.: Scaling regimes of composite rainfall time series, Tellus, 45A, 289-298, 1993.

Fraedrich, K., Ziehmann, C., and Sielmann, F.: Estimates of spatial degrees of freedom, J. Climate, 8, 361-369, 1994.

Fraedrich, K., Kirk, E., and Lunkeit, F.: PUMA: Portable University Model of the Atmosphere, DKRZ-Report No. 16, Deutsches Klima Rechenzentrum, Hamburg, 1998, available from: Deutsches Klima Rechenzentrum, Bundesstr. 55, D-20146 Hamburg, Germany; see also http://dome.dkrz.de/puma

Franzke, Chr., Fraedrich, K., and Lunkeit, F.: Low frequency variability in a simplified atmospheric GCM: Storm track induced "spatial resonance", Q. J. R. Meteorol. Soc., 126, 2691-2708, 2000.

Fraser, A. M. and Swinney, H. L.: Independent coordinates for strange attractors from mutual information, Phys. Rev. A, 33, 1134-1139, 1986.

Frisius, T., Lunkeit, F., Fraedrich, K., and James, I.: Storm-track organisation and variability in a simplified atmospheric global circulation model, Q. J. R. Meteorol. Soc., 124, 1019-1043, 1998.

Grassberger, P. and Procaccia, I.: Measuring the strangeness of strange attractors, Physica D, 9, 189-208, 1983.

Hegger, R., Kantz, H., and Schreiber, T.: Practical implementation of nonlinear time series methods: The TISEAN package, 1998, available at http://www.mpipks-dresden.mpg.de/ tisean.

Hoskins, B. and Simmons, A.: A multi-layer spectral model and the semi-implicit method, Q. J. R. Meteorol. Soc., 101, 637-655, 1975.

James, I. N. and James, P. M.: Ultra-low-frequency variability in a simple atmospheric circulation model, nature, 342, 53-55, 1989.

James, I. N., and James, P. M.: Spatial structure of ultra-lowfrequency variability of the flow in a simple atmospheric cir- culation model, Q. J. R. Meteorol. Soc., 118, 1211-1233, 1992.

James, P. M., Fraedrich, K., and James, I. N.: Wave-zonal flow interaction and ultra-low-frequency variability in a simplified global circulation model, Q. J. R. Meteorol. Soc., 120, 10451067, 1994.

James, I. N., and Dodd, J. D.: A mechanism for the low-frequency variability of the mid-latitude troposphere, Q. J. R. Meteorol. Soc., 122, 1197-1210, 1996.

Kantz, H. and Schreiber, T.: Nonlinear time series analysis, Cambridge University Press, Cambridge, 1997.

Kaulakys, B. and Meškauskas, T.: Modelling $1 / f$ noise, Phys. Rev. B, 58, 7013-7019, 1998.

Kennel, M. B., Brown, R., and Abarbanel, H. D. I.: Determining embedding dimension for phase-space reconstruction using a geometrical construction, Phys. Rev. A, 45, 3403-3411, 1992.

Koscielny-Bunde, E., Bunde, A., Havlin, S., Roman, H. E., Goldreich, Y., and Schellnhuber, H.-J.: Indication of a universal persistence law governing atmospheric variability, Phys. Rev. Lett., 81, 729-732, 1998.

Kushnir, Y.: Retrograding wintertime low-frequency disturbances over the North Pacific Ocean, J. Atmos. Sci., 47, 2115-2140, 1987.

Lorenz, E. N.: Deterministic nonperiodic flow, J. Atmos. Sci., 20, 130-141, 1963.

Lorenz, E. N.: Atmospheric predictability as revealed by naturally occurring analogues, J. Atmos. Sci., 26, 636-646, 1969.

Nicolis, C. and Nicolis, G.: Is there a climate attractor?, nature, 311, 529-532, 1984.

Peng, C.-K., Buldyrev, S. V., Havlin, S., Simons, M., Stanley, H. E., and Goldberger, A. L.: Mosaic organization of DNA nucleotides, Phys. Rev. E, 49, 1685-1689, 1994.

Perrier, V., Philipovitch, T., and Basdevant, C.: Wavelet spectra compared to Fourier spectra, J. Math. Phys., 36, 1506-1519, 1995.

Riehl, H., Yeh, T. C., and La Seur, N. E.: A study of variations of the general circulation, J. Meteorol., 7, 181-194, 1950.

Schreiber, T.: Interdisciplinary application of nonlinear time series methods, Physics Reports, 308, 1-64, 1999.

Sura, P., Lunkeit, F., and Fraedrich, K.: Decadal variability in a simplified wind-driven ocean model, J. of Phys. Oceanogr., 30, 1917-1930, 2000.

Takens, F.: Detecting strange attractors in turbulence, in: Dynamical systems and turbulence, Lecture Notes in Mathematics, Vol. 898, Springer-Verlag, New York, 366-381, 1981.

Torrence, C. and Compo, G. P.: A practical guide to wavelet analysis, Bull. Amer. Meteor. Soc., 79, 61-78, 1998.

Toth, Z.: Dimension estimates of the Northern hemisphere circulation phase space, Tellus, 47A, 457-472, 1995.

Yano, J.-I., Fraedrich, K., and Blender, R.: Tropical convective variability as 1/f-noise, J. Climate, 14, 3608-3616, 2001. 